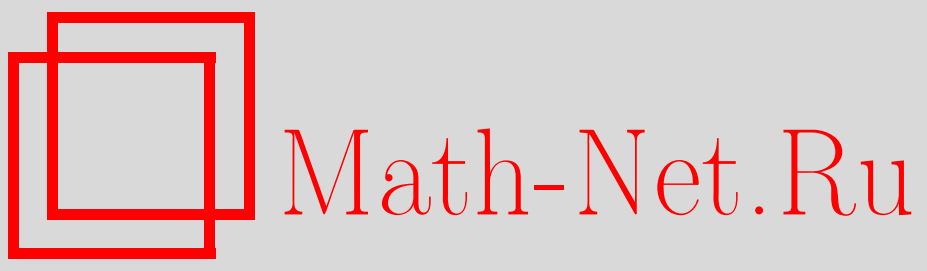

В. В. Кварацхелия, Некоторые неравенства, связанные с матрицами Адамара, Функи. анализ и его прил., 2002, том 36, выпуск 1, 81-85

DOI: https://doi.org/10.4213/faa181

Использование Общероссийского математического портала MathNet.Ru подразумевает, что вы прочитали и согласны с пользовательским соглашением

http://www.mathnet.ru/rus/agreement

Параметры загрузки:

IP : 54.172 .240 .79

26 апреля 2023 г., $17: 25: 47$

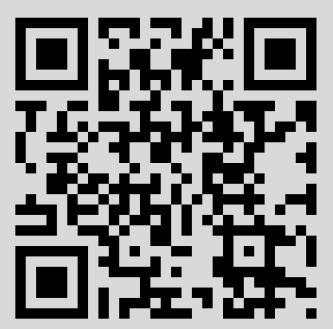


СледСтвиЕ 2. Функциия $K(x, y)$ удовлетворяет дифференциильному уравнению

$$
K_{x}=(1-2 x) K_{y y}-x y K_{y y y} .
$$

Наконец, соотношение теоремы позволяет получить числа $K_{n}^{l}$, где $n \leqslant 0$.

\begin{tabular}{|c|c|c|c|c|c|}
\hline \multicolumn{1}{|c|}{$K_{n}^{l}$} & $l=1$ & $l=2$ & $l=3$ & $l=4$ & $l=5$ \\
\hline$n=0$ & 1 & 0 & 0 & 0 & 0 \\
\hline$n=-1$ & 1 & 0 & 0 & 0 & 0 \\
\hline$n=-2$ & $?$ & 1 & 0 & 0 & 0 \\
\hline$n=-3$ & $?$ & $?$ & 2 & 0 & 0 \\
\hline$n=-4$ & $?$ & $?$ & $?$ & 6 & 0 \\
\hline$n=-5$ & $?$ & $?$ & $?$ & $?$ & 24 \\
\hline
\end{tabular}

Можно считать, что $K_{0}^{l}$ - это количество компонент связности пространства многочленов степени 0 c $l$ краевыми точками, в которых многочлен принимает разные значения. В этом случае все точки прямой критические, и соответственно критическое значение совпадает с краевыми. Что обозначают числа $K_{n}^{l}$ для отрицательных $n$, автору неизвестно.

Заметим, что с некоторого момента в каждой строке таблицы стоят нули. Сформулируем это утверждение:

СледСТВИЕ 3. Пусть $n \leqslant-1 ; \operatorname{moг\partial a~} K_{n}^{l}=0$ для $l>-n, a K_{n}^{-n}=(-n-1)$ !. Кроме того, $K_{0}^{l}=0$ при $l>1$.

Доказательство опирается на теорему и проводится индукцией по $n$.

\title{
ЛИТЕРАТУРА
}

1. Arnold V. I. Duke Math. J., 63, No. 2, 537-555 (1991). 2. Arnold V. I. J. Algebraic Geom., 1, No. 2, 197-214 (1992).

Московский государственный университет, механико-математический факультет
Поступило в редакцию 26 сентября 2000 г.

\section{Некоторые неравенства, связанные с матрицами Адамара}

\author{
(C) 2002. В. В. КВАРАЦХЕЛИЯ
}

В настоящей работе вводится числовая характеристика матрицы Адамара (Сильвестра), которая играет важную роль при изучении сходимости рядов в банаховых пространствах (см. [1-3]). Здесь приводятся оценки этой характеристики, которые, в частности, можно интерпретировать как конкретные максимальные неравенства в банаховых пространствах с субсимметрическим базисом. Как нам кажется, ресурс введенной характеристики далеко не исчерпан, по крайней мере с точки зрения изучения сходимости рядов. 
Матрицей Адамара называется квадратная матрица порядка $n$, такая, что ее элементы суть \pm 1 и любые две ее строки (столбцы) ортогональны (см., например, $\left[4\right.$, c. 283] и [5, с. 52]). Матрицу Адамара порядка $n$ будем обозначать через $\mathscr{H}_{n}=$ $\left[h_{k i}^{n}\right]$. Легко видеть, что если матрица Адамара существует, то ее порядок равен 1 или 2 либо делится на 4. Адамаром была высказана гипотеза, что для любого $n$, кратного 4, существует матрица Адамара порядка $n$. Насколько нам известно, вопрос о справедливости гипотезы Адамара остается открытым. Обозначим через $\mathscr{N}_{H}$ множество всех натуральных чисел $n$, для которых существует матрица Адамара порядка $n$. Например, $2^{n} \in \mathscr{N}_{H}$ для всех $n=1,2, \ldots$.

Матрица Сильвестра - частный случай матрицы Адамара и определяется следующими рекуррентными соотношениями (см. [2, с. 52]):

$$
\mathscr{S}^{(1)}=\left[\begin{array}{cc}
1 & 1 \\
1 & -1
\end{array}\right], \quad \mathscr{S}^{(n)}=\left[\begin{array}{cc}
\mathscr{S}^{(n-1)} & \mathscr{S}^{(n-1)} \\
\mathscr{S}^{(n-1)} & -\mathscr{S}^{(n-1)}
\end{array}\right], \quad n=2,3, \ldots
$$

Ясно, что $\mathscr{S}^{(n)}$ действительно является матрицей Адамара порядка $2^{n}$.

Используемые здесь понятия и их свойства можно найти, например, в [6-8].

Пусть $\mathscr{S}^{(n)}=\left[s_{k i}^{(n)}\right], n=1,2, \ldots,-$ матрица Сильвестра и $X-$ банахово пространство с нормированным базисом $\left(\varphi_{k}\right)$. Для произвольного данного $n$ рассмотрим следующий функционал:

$$
\varrho^{(n)}(m)=\left\|\sum_{i=1}^{2^{n}}\left(\sum_{k=1}^{m} s_{k i}^{(n)}\right) \varphi_{i}\right\|, \quad m=1, \ldots, 2^{n} .
$$

Выражение $\varrho^{(n)}(m)$, очевидно, зависит от $X$, от нормы в $X$ и от выбора базиса $\left(\varphi_{k}\right)$ в $X$.

Положим

$$
\varrho^{(n)}=\max _{1 \leqslant m \leqslant 2^{n}} \varrho^{(n)}(m)
$$

Функционал $\varrho^{(n)}(m)$ можно выразить и следующим образом. Пусть $a_{k}=$ $\sum_{i=1}^{2^{n}} s_{k i}^{(n)} \varphi_{i}, k=1, \ldots, 2^{n}$. Тогда $\varrho^{(n)}(m)=\left\|\sum_{k=1}^{m} a_{k}\right\|$. Если $\left(\varphi_{k}\right)-$ безусловный базис с единичной константой безусловности, то очевидно, что $\left\|a_{k}\right\|=\lambda\left(2^{n}\right)$ для всех $k=1, \ldots, 2^{n}$ и $\varrho^{(n)} \leqslant \lambda\left(2^{n}\right) 2^{n} \leqslant 2^{2 n}$, где $\lambda(n)=\left\|\sum_{k=1}^{n} \varphi_{k}\right\|, n=$ $1,2, \ldots$.

Доказано, что в пространстве $l_{p}, 1 \leqslant p<\infty$, выполняется неравенство $\varrho^{(n)} \leqslant$ $n 2^{n}$, и с помощью этой оценки в [3] строятся нетривиальные условно сходящиеся ряды в $l_{p}, 1 \leqslant p<2$. Следующая теорема дает аналогичную оценку для $\varrho^{(n)}$ в случае общих банаховых пространств с субсимметрическим базисом.

Tеорема 1. Пусть $\mathscr{S}^{(n)}=\left[s_{k i}^{(n)}\right], n=1,2, \ldots,-$ матрица Сильвестра, пусть $\varrho^{(n)}$ - выражение, определенное равенством (2), и пусть нормированньй базис $\left(\varphi_{k}\right)$ банахова пространства $X$, участвующий в определении выражения $\varrho^{(n)}$, является субсимметрическим с единичной константой субсимметричности. Tогда

$$
\varrho^{(n)} \leqslant\left\{1+\sum_{j=1}^{n} 2^{-j} \lambda\left(2^{j-1}\right)\right\} 2^{n}, \quad n=1,2, \ldots
$$


СледствиЕ 2. Пусть банахово пространство Х удовлетворяет требованиям теоремь 1. Тогда

$$
\varrho^{(n)} \leqslant \lambda(n) 2^{n} .
$$

Пусть $X$ - произвольное банахово пространство (не обязательно с базисом), $x_{1}, \ldots, x_{2^{n}}$ - последовательность произвольных элементов из единичной сферы пространства $X$ и $\mathscr{S}^{(n)}$ - матрица Сильвестра порядка $2^{n}, n=1,2, \ldots$ По аналогии с формулой $(1)$ пусть $\hat{\varrho}^{(n)}(m)=\left\|\sum_{i=1}^{2^{n}}\left(\sum_{k=1}^{m} s_{k i}^{(n)}\right) x_{i}\right\|, m=1, \ldots, 2^{n}$, и пусть $\hat{\varrho}^{(n)}=\max _{1 \leqslant m \leqslant 2^{n}} \hat{\varrho}^{(n)}(m)$.

СледСтВИЕ 3. Имеет место неравенство

$$
\hat{\varrho}^{(n)} \leqslant n 2^{n} .
$$

СледСТвиЕ 4. Пусть $X$ - банахово пространство типа $p, p>1$, с нормированным субсимметрическим базисом $\left(\varphi_{k}\right)$ с единичной константой субсимметричности. Тогда справедлива оценка

$$
\varrho^{(n)} \leqslant c 2^{n},
$$

где константа $c \geqslant 1$ зависит только от пространства $X$.

Таким образом, в пространствах типа $p, p>1\left(\right.$ а также в $\left.c_{0}\right), \sup _{n} \varrho^{(n)} / 2^{n}$ $<\infty$. Следующее утверждение показывает, что в пространстве $l_{1}$ это не так.

ПрЕДЛОЖЕНИЕ 5. В пространстве $l_{1}$ относительно естественного базиса имеет место равенство

$$
\varrho^{(n)}=\max _{1 \leqslant m \leqslant 2^{n}} \varrho^{(n)}(m)=(3 n+7) 2^{n} / 9+2(-1)^{n} / 9, \quad n \geqslant 1 .
$$

Более того, для всех $n$ максимум достигается в точке $m_{n}=\left(2^{n+1}+(-1)^{n}\right) / 3$, и если $n \geqslant 3$, то тот же максимум достигается также и в точке $m_{n}^{\prime}=$ $\left(5 \cdot 2^{n-1}+(-1)^{n-1}\right) / 3$.

Оценим $\varrho^{(n)}$ снизу.

ПрЕДЛОЖЕНИЕ 6. Если банахово пространство X удовлетворяет требованиям теоремь 1, то

$$
\varrho^{(n)} \geqslant \max \left\{(n+2) \lambda\left(2^{n}\right) / 6,2^{n}\right\}, \quad n=1,2, \ldots .
$$

Если базис $\left(\varphi_{k}\right)$ симметрический, то делитель 6 в (3) можно заменить на 3.

Заметим, что помимо $l_{1}$ существуют и другие банаховы пространства, в кото-

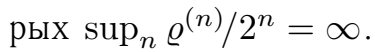

Естественно возникает вопрос, как изменяются указанные здесь результаты, полученные для матриц Сильвестра, при переходе к общим матрицам Адамара. Обозначим через $\mathscr{H}_{n}^{\text {all }}$ множество всех матриц Адамара порядка $n, n \in \mathscr{N}_{H}$ (ясно, что если $n=2^{j}, j=1,2, \ldots$, то $\mathscr{H}_{n}^{\text {all }}$ содержит и матрицы Сильвестра порядка $\left.2^{j}\right)$. Для матрицы Адамара $\mathscr{H}_{n}=\left[h_{k i}^{n}\right]$ аналогично введем числовую характеристику $\varrho \mathscr{H}_{n}(m)=\left\|\sum_{i=1}^{n}\left(\sum_{k=1}^{m} h_{k i}^{n}\right) \varphi_{i}\right\|, m=1, \ldots, n$, где $\left(\varphi_{i}\right)-$ нормированный базис банахова пространства $X$. Если положить $a_{k}=\sum_{i=1}^{n} h_{k i}^{n} \varphi_{i}$, то, очевидно, что $\varrho_{\mathscr{H}_{n}}(m)=\left\|\sum_{k=1}^{m} a_{k}\right\|$. Ясно, что если $\left(\varphi_{k}\right)-$ безусловный базис с единичной константой безусловности, то $\max _{1 \leqslant m \leqslant n} \varrho_{\mathscr{H}_{n}}(m) \leqslant \lambda(n) n \leqslant n^{2}$ для всех $\mathscr{H}_{n} \in \mathscr{H}_{n}^{\text {all }}$. 


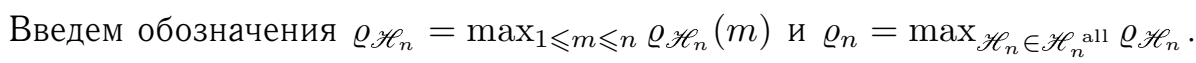

ЗАмечАние 7. Характеристику $\varrho_{\mathscr{H}_{n}}=\varrho\left(\mathscr{H}_{n}\right)$ можно интерпретировать как норму матрицы $\mathscr{H}_{n}$. В самом деле, обозначим через $M_{n}, n \in \mathscr{N}_{H}$, векторное пространство всех квадратных матриц порядка $n$, и пусть $X$ - банахово пространство с нормированным базисом $\left(\varphi_{i}\right)$. Очевидно, что $\mathscr{H}_{n}^{\text {all }} \subset M_{n}$. Пусть $\mathscr{T}_{n}=\left[t_{k i}^{n}\right]$ $\in M_{n}$ - произвольная матрица, и пусть $\varrho\left(\mathscr{T}_{n}\right)=\max _{1 \leqslant m \leqslant n}\left\|\sum_{i=1}^{n}\left(\sum_{k=1}^{m} t_{k i}^{n}\right) \varphi_{i}\right\|$. Легко видеть, что $\varrho$ является нормой в пространстве $M_{n}$ и относительно этой нормы $M_{n}$ является банаховым пространством.

Следующая теорема дает оценку снизу для выражения $\varrho_{n}$.

Теорема 8. Пусть $X-$ банахово пространство с нормированным безусловным базисом с единичной константой безусловности. Тогда

$$
\varrho_{n} \geqslant \max \{(1 / \sqrt{2}) \lambda(n) \sqrt{n}, n\} \quad \text { для всех } n \in \mathscr{N}_{H} .
$$

Сформулируем аналог теоремы 1 в случае матриц Адамара.

Теорема 9. Пусть $X-$ банахово пространство с нормированным субсимметрическим базисом с единичной константой субсимметричности. Тогда

$$
\varrho_{n} \leqslant \lambda([\sqrt{n}]+1) n
$$

для всех $n \in \mathscr{N}_{H}$, где $[\sqrt{n}]$ - целая часть числа $\sqrt{n}$.

Следующее предложение уточняет теорему 9 в случае $l_{p}, 1 \leqslant p<\infty$.

ПреДЛОЖениЕ 10. В пространстве $l_{p}, 1 \leqslant p<\infty$, относительно естественного базиса для всех $n \in \mathscr{N}_{H}$ справедливо неравенство

$$
\varrho_{n} \leqslant \max \left\{n^{(p+2) / 2 p}, n\right\} .
$$

Для матриц Сильвестра из предложений 6 и 10 вытекает

Следствие 11. В пространстве $l_{p}, p \geqslant 2$, относительно естественного базиса

$$
\varrho^{(n)}=2^{n} .
$$

Пусть $X$ - банахово пространство (не обязательно с базисом), $x_{1}, \ldots, x_{n}$ - последовательность произвольных элементов из единичной сферы пространства $X$ и $\mathscr{H}_{n} \in \mathscr{H}_{n}^{\text {all }}, n \in \mathscr{N}_{H}$. По аналогии введенными выше выражениями рассмотрим выражения $\hat{\varrho}_{\mathscr{H}_{n}}(m)=\left\|\sum_{i=1}^{n}\left(\sum_{k=1}^{m} h_{k i}^{n}\right) x_{i}\right\|, m=1, \ldots, n$,

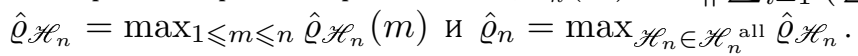

СледствиЕ 12. Для всех $n \in \mathscr{N}_{H}$ справедливо неравенство

$$
\hat{\varrho}_{n} \leqslant n \sqrt{n} \text {. }
$$

ЗАмЕчАниЕ 13. Теорему 9 можно перефразировать в терминах максимального неравенства. Пусть $\mathscr{H}_{n}=\left[h_{k i}^{n}\right]-$ произвольная матрица Адамара порядка $n \in \mathscr{N}_{H}$, и пусть $a_{k}=\sum_{i=1}^{n} h_{k i}^{n} \varphi_{i}, k=1, \ldots, n$, где $\left(\varphi_{i}\right)-$ нормированный субсимметрический базис банахова пространства $X$ с единичной константой субсимметричности. Тогда

$$
\max _{1 \leqslant m \leqslant n}\left\|\sum_{k=1}^{m} \vartheta_{k} a_{k}\right\| \leqslant \lambda([\sqrt{n}]+1) n
$$




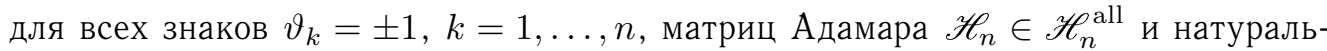
ных чисел $n \in \mathscr{N}_{H}$.

С помощью $\varrho^{(n)}$ и $\varrho_{n}$ можно охарактеризовать пространства, изоморфные $l_{1}$.

Теорема 14. Пусть $X-$ банахово пространство с нормированньлм субсимметрическим базисом $\left(\varphi_{k}\right)$ с единичной константой субсимметричности. Следующие утверждения эквивалентнь:

(i) пространство $X$ изоморфно $l_{1}$;

(ii) существует константа $\varepsilon>0$, такая, что $\varrho^{(n)} /\left(n 2^{n}\right) \geqslant \varepsilon$ для всех $n=$ $1,2, \ldots$, где в не зависит от $n$;

(iii) существует константа $\delta>0$, такая, что $\varrho_{n} /(n \sqrt{n}) \geqslant \delta$ для всех $n \in \mathscr{N}_{H}$, где $\delta$ не зависит от $n$.

Автор выражает глубокую признательность проф. Н. Н. Вахания и С. А. Чобаняну за внимание и обсуждение полученных результатов.

\section{ЛИТЕРАТУРА}

1. Vakhania N., Kvaratskhelia V. Bull. Georgian Acad. Sci., 160, No. 2, 201-203 (1999). 2. Vakhania N., Kvaratskhelia V. Bull. Georgian Acad. Sci., 162, No. 2, 199-202 (2000). 3. Vakhania N., Kvaratskhelia V. Bull. Georgian Acad. Sci., 163, No. 3 (2001). 4. Xoлл M. Комбинаторика. Мир, М., 1970. 5. Мак-Вильямс Ф. Дж., Слоэн Н. Дж. А. Теория кодов, исправляющих ошибки. Связь, М., 1979. 6. Lindenstrauss J., Tzafriri L. Classical Banach spaces I. Sequence spaces. Springer-Verlag, Berlin-Heidelberg-New York, 1977. 7. Diestel J., Jarchow H., Tonge A. Absolutely summing operators. Cambridge University Press, Cambridge, 1995. 8. Day M. M. Normed linear spaces. Springer-Verlag, BerlinHeidelberg-New York, 1973.

Институт вычислительной математики им. Н. И. Мусхелишвили АН Грузии

Поступило в редакцию 27 октября 2000 г. В переработанном виде 27 августа 2001 г.

УДК 517.982

\section{Строго сингулярные вложения*}

(c) 2002. С. Я. Новиков, Е. М. Семенов, Ф. Л. ЭРНАНДЕС

Оператор $A$, действующий из банахова пространства $E$ в банахово пространство $F$, называется строго сингулярным или оператором Kaто, если никакое его сужение на бесконечномерное подпространство $B \subset E$ не является изоморфизмом $[1, \mathrm{Sec}$. 2.c]. Оператор $A$, действующий из банаховой решетки $E$ в банахово пространство $F$, называется дизъюнктно строго сигулярным, если никакое его сужение на бесконечномерное подпространство, порожденное системой дизъюнктных элементов, не является изоморфизмом [2].

Банахово пространство $E$ измеримых на $[0,1]$ функций называется перестановочно-инвариантным (г.і.) или симметричным, если

1) из неравенства $|x(t)| \leqslant|y(t)|$ для всех $t \in[0,1]$ и $y \in E$ вытекает, что $x \in E$ и $\|x\|_{E} \leqslant\|y\|_{E}$;

* Работа второго автора была частично поддержана РФФИ, грант 98-01-00044, и фондом «Университеты России», грант 3667, а третьего автора - DGIES (Испания), грант PB 97-0240. 